\title{
THEORETICAL PEARLS
}

\section{Enumerators of lambda terms are reducing}

\author{
HENK BARENDREGT
}

Faculty of Mathematics and Computer Science, Catholtc University of Nijmegen, The Netherlands

\begin{abstract}
A closed $\lambda$-term $\mathbf{E}$ is called an enumerator if

$$
\forall M \in \Lambda^{0} \exists n \in \mathbb{N} \boldsymbol{C}^{\top} n^{\urcorner}={ }_{\beta} M .
$$

Here $\Lambda^{0}$ is the set of closed $\lambda$-terms, . is the set of natural numbers and the ${ }^{\ulcorner} n$ ? are the Church's numerals $\lambda f x . f^{n} x$. Such an $\mathbf{E}$ is called reducing if, moreover

$$
\left.\forall M \in \Lambda^{0} \exists n \in \mathbb{N} E^{\top} n\right\urcorner \rightarrow{ }_{\beta} M .
$$

An ingenious recursion theoretic proof by Statman will be presented, showing that every enumerator is reducing. I do not know any direct proof.
\end{abstract}

\section{Introduction}

Remember that in Barendregt (1991) a simple proof of the existence of a selfinterpreter $E \in \Lambda^{0}$ was given. Such an $E$ satisfies

$$
\forall M \in \Lambda^{0} \mathrm{E}^{\mathrm{r}} M^{\mathrm{\urcorner}}={ }_{\beta} M .
$$

The first construction of a self-interpreter is due to Kleene (1936), and I presented another one due to P. de Bruin. Such an $E$ is automatically an enumerator. Inspection of the details of the construction of $E$ by Kleene (1936) or by P. de Bruin shows that these $\mathbf{E}$ are in fact reducing enumerators.

In my thesis (Barendregt, 1971) I constructed as application a universal generator, that is, a term reducing to terms of arbitrary complexity.

\section{Definition 1.1}

A term $U \in \Lambda$ is called a universal generator iff

$$
\forall M \in \Lambda \exists N \in \Lambda\left[U \rightarrow{ }_{\beta} N \& M \text { subterm of } N\right] .
$$

\section{Proposition 1.2}

There exists a universal generator $U \in \Lambda^{0}$.

Proof

If $\mathbf{E}$ is a reducing enumerator, then one can take $U \equiv F^{\mathrm{r}} 0^{\urcorner}$with

$$
\left.F\ulcorner n\urcorner \rightarrow_{\beta}\left[\mathrm{E}^{\top} n\right\urcorner, F\ulcorner n+1\urcorner\right],
$$


where $[-,-]$ is a pairing in the $\lambda$-calculus. Indeed, one then has

$$
\begin{aligned}
U & \left.\rightarrow{ }_{\beta} F^{\ulcorner} 0\right\urcorner \\
& \left.\rightarrow{ }_{\beta}\left[E^{\ulcorner} 0\right\urcorner, F\ulcorner 1\urcorner\right] \\
& \left.\left.\left.\rightarrow{ }_{\beta}\left[E^{\ulcorner} 0\right\urcorner,\left[E^{\ulcorner} 1\right\urcorner, F^{\ulcorner} 2\right\urcorner\right]\right] \\
& \ldots \\
& \left.\left.\left.\rightarrow \rightarrow_{\beta}\left[E^{\ulcorner} 0\right\urcorner,\left[E^{\ulcorner} 1\right\urcorner,\left[E^{\ulcorner} 2\right\urcorner,[\ldots]\right]\right]\right] \\
& \ldots
\end{aligned}
$$

Since the $\left.\mathrm{E}^{\ulcorner} n\right\urcorner$ collectively reduce to all $M \in \Lambda^{0}$ and any $N \in \Lambda$ is a subterm of some closed term, it follows that $U$ is a universal generator.

$F$ can be constructed easily from $E$ using the fixed-point combinatory and a $\lambda$ defining term for the successor function. (For example,

$$
U \equiv(\lambda a b . b(a a b))(\lambda a b . b(a a b))(\lambda f x z . z(\mathbf{E} x)(f(\lambda b c . b(x b c))))(\lambda b c . c)
$$

works.)

A short universal generator has been constructed in Mulder (1990): in one of the propositions (stellingen) accompanying the thesis he constructed

$$
\begin{aligned}
U \equiv & (\lambda k p y \cdot y(\lambda l \cdot p k(p(\lambda x y z \cdot x z(y z)) \\
& (y(\lambda f x y z \cdot z k(\lambda h t \cdot x(f(p h y)(p h y) l))(\lambda u v \cdot p(h u)(f v y t))) k k l)))) \\
& (\lambda x y \cdot x)(\lambda x y z f \cdot f x y)(\lambda f \cdot(\lambda x \cdot x x)(\lambda x \cdot f(x x))) .
\end{aligned}
$$

The fact that the given enumerators are reducing brought me to the following:

\section{Conjecture 1.3}

Every enumerator is reducing.

Some vague evidence for the conjecture is this. If $\mathbf{E}$ has to make every $M \in \Lambda^{0}$ by having $\left.\mathbb{E}^{\Gamma} n\right\urcorner={ }_{\beta} M$ for some $n \in \mathbb{N}$, then the only way to do this is to construct every $M \in \Lambda^{0}$ by a reduction from $\left.E^{\top} n\right\urcorner$ for an appropriate $n$. This is plausible, since the collection

$$
B_{M}=\left\{N \in \Lambda^{0} \mid M={ }_{\beta} N\right\}
$$

is undecidable. It seems easier to make the $E^{\ulcorner} n^{\urcorner}$reduce to all members of all $B_{M}$ than to just some of them.

Of course, this intuition is far from being a proof. I explained my conjecture to Rick Statman in 1983 and in 1987 he settled it in the positive. In fact, as we will see, he proved something much more general.

\section{Proof of the conjecture}

If $\psi$ is a partial recursive function, then $\psi(n) \downarrow$ means that $\psi(n)$ is defined and $\psi(n) \uparrow$ means that $\psi(n)$ is undefined. A set $A \subseteq \mathbb{N}$ is called recursively enumerable (r.e.) if for some partial recursive $\psi: \mathbb{N} \rightarrow \mathbb{N}$ one has $A=\operatorname{dom}(\psi)$, i.e. $\forall n \in \mathbb{N}[n \in A \Leftrightarrow \psi(n) \downarrow]$. In 
the following the reader is supposed to know some elementary properties of r.e. sets. For example, that if $A$ and its complement are both r.e., $A$ is recursive; moreover, that there exists a set $K \subseteq \mathbb{N}$ that is r.e. but not recursive.

\section{Lemma 2.1}

For every $M \in \Lambda$ there exists an $M_{1} \in \Lambda$ in $\beta$-nf such that $M_{1} \mid \rightarrow_{\beta} M$. Here $\mathbf{I} \equiv \lambda x$.x.

\section{Proof}

By induction on the structure of $M$ we define $M_{1}$ in the following table:

\begin{tabular}{ll}
\hline \hline$M$ & $M_{1}$ \\
\hline$x$ & $\lambda z . z x$ \\
$P Q$ & $\lambda z . z P_{1} z\left(z Q_{1} z\right)$ \\
$\lambda x . P$ & $\lambda z x . z P_{1} z$ \\
\hline
\end{tabular}

Then by induction it follows that $M_{1} \mathrm{I} \rightarrow \rightarrow_{\beta} M$.

Remember that a term $M \in \Lambda$ is of order 0 if for no $P \in \Lambda$ one has $M={ }_{\beta} \lambda x$.P. For example $(\lambda x . x x)(\lambda x . x x)$ is of order 0 .

\section{Lemma 2.2}

(i) For every partial recursive function $\psi$ there is a term $F \in \Lambda^{0}$ such that for all $n \in \mathbb{N}$ one has

$$
\begin{aligned}
& \left.\psi(n) \downarrow \Rightarrow F^{\ulcorner} n\right\urcorner={ }_{\beta}\ulcorner\psi(n)\urcorner \\
& \left.\psi(n) \uparrow \Rightarrow F^{\ulcorner} n\right\urcorner \text { is of order } 0 .
\end{aligned}
$$

(ii) let $K \subseteq \mathbb{N}$ be an r.e. set. Then for some $P_{K} \in \Lambda^{0}$ one has for all $n \in \mathbb{N}$

$$
\begin{aligned}
& n \in K \Rightarrow P_{K} \Gamma n^{\urcorner} \rightarrow{ }_{\beta} \mid ; \\
& n \notin K \Rightarrow P_{K} \Gamma_{n\urcorner} \text { is of order } 0 .
\end{aligned}
$$

\section{Proof}

(i) Inspection of the usual proof of the $\lambda$-definability of the partial recursive functions shows that in case the function is undefined on an argument the representing $\lambda$-term is of order 0 on the corresponding numerical. (One of the next 'Pearls in Theory' will be devoted to possible representations of 'undefined'.)

(ii) Let $K=\operatorname{dom}(\psi)$. Let $F \lambda$-define $\psi$. Then take $P_{K} \equiv \lambda c . F c l l$. (Remember that for Church's numerals one has $r n$ ㄱI $={ }_{\beta} I$.]

Theorem 2.3 (Statman, 1987)

Let $\mathscr{A} \subseteq \Lambda^{0}$ (after coding) be an r.e. set. Suppose

Then

$$
\forall M \in \Lambda^{0} \exists N \in \mathscr{A} N={ }_{\beta} M .
$$

$$
\forall M \in \Lambda^{0} \exists N \in \mathscr{A} N \rightarrow_{\beta} M .
$$




\section{Proof}

Assume (1). Suppose towards a contradiction that (2) does not hold, i.e. for some $M_{0} \in \Lambda^{0}$

$$
\forall N \in \mathscr{A} N \nrightarrow{ }_{\beta} M_{0} .
$$

Using Lemma 2.1 construct a term $M_{1}$ in $\beta$-nf such that $M_{1} I \rightarrow_{\beta} M_{0}$. Define a predicate $R$ on $\mathbb{N}$ as follows:

$$
\left.R(n) \Leftrightarrow \exists N \in \mathscr{A} \exists Q \in \Lambda\left[P^{\ulcorner} n^{\prime} \rightarrow_{\beta} Q \& N \rightarrow_{\beta} Q M_{1}\right]\right],
$$

where $P=P_{K}$ as in Lemma 2.2 for some non-recursive r.e. set $K$. Note that $R$ is an r.e. predicate. Claim

$$
R(n) \Leftrightarrow n \notin K .
$$

As to $(\Rightarrow)$, suppose $R(n)$, i.e. for some $N \in \mathscr{A}$ and $Q \in \mathscr{A}$ one has

$$
P\ulcorner n\urcorner \rightarrow_{\beta} Q \text { and } N \rightarrow_{\beta} Q M_{1} \mathbf{I} \text {. }
$$

If $n \in K$, then $\mathrm{I}={ }_{\beta} P{ }^{\ulcorner} n^{\top}={ }_{\beta} Q$, so by the Church-Rosser theorem $Q \rightarrow \rightarrow_{\beta} \mathrm{I}$ and therefore $N \rightarrow_{\beta}\left|M_{1}\right| \rightarrow_{\beta} M_{0}$, contracting (2). Therefore $n \notin K$ and we are done. As to $(\Leftarrow)$, suppose $n \notin K$. Then $P\ulcorner n\urcorner$ is of order 0 . By (1) there is an $N \in \mathscr{A}$ such that $N={ }_{\beta} P^{\ulcorner} n^{\top} M_{1}$ I. By the Church-Rosser theorem there is a common reduct $L$ of $N$ and $P\ulcorner n\urcorner M_{1} \mid$. Since $P\ulcorner n\urcorner$ is of order 0 and $M_{1}, I$ are in nf one must have $L \equiv Q M_{1} \mid$ with $P\ulcorner n\urcorner \rightarrow_{\beta} Q$. Therefore $R(n)$.

From the claim it follows that the complement of $K$ is r.e., hence recursive (since $K$ is itself r.e.) contradicting the choice of $K$.

From the theorem the conjecture follows immediately by taking $\left.\mathscr{A}=\left\{\mathbb{E}^{\ulcorner} n\right\urcorner \mid n \in \mathbb{N}\right\}$.

From the proved conjecture I mistakenly concluded that every self-interpreter in the $\lambda$-calculus is reducing in the sense that

$$
\forall M \in \Lambda^{0} \mathrm{Er}^{\mathrm{N}} M \rightarrow_{\beta} M .
$$

But this does not follow. Do you see why? Moreover, that this is not true was pointed out to me by Peter de Bruin, who provided a counterexample. Can you construct one?

\section{References}

Barendregt, H. P. 1971. Some extensional term models for lambda calculi and combinatory logics. $\mathrm{PhD}$ thesis, Utrecht University.

Barendregt, H. P. 1991. Theoretical pearls: Self-interpretation in lambda calculus. J. Functional Programming, 1 (2), 229-234.

Kleene, S. C. 1936. $\lambda$-definability and recursiveness. Duke Math. J., 2, 340-353.

Mulder, J. C. 1990. Case studies in process specification and verification. $\mathrm{PhD}$ thesis, University of Amsterdam.

Statman, R. 1987. Two recursion theoretic problems in lambda calculus. Manuscript, Department of Mathematics, Carnegie Mellon University, Pittsburgh, PA. 